\title{
氮素对内蒙古典型草原羊草种群的影响
}

\author{
潘庆民 白永飞 韩兴国 杨景成 \\ (中国科学院植物研究所内蒙古草原生态系统定位研究站, 北京 100093)
}

\begin{abstract}
摘 要 为了研究氮素对内蒙古典型草原植物种群的影响, 在中国科学院内蒙古草原生态系统定位研究站, 实施 了长期的氮素添加试验。就两年来不同梯度氮素处理对羊草(Leymus chinensis)种群的影响进行了分析。结果表明, 氮素对羊草种群具有显著的调节效应, 随着氮素梯度的增加, 羊草种群密度、种群高度、地上生物量、地下生物量、 总生物量均显著增加, 羊草种群地下生物量/地上生物量比值逐渐降低。氮素对羊草种群构件的生物量分配有显 著影响, 随着氮素梯度的增加, 羊草种群生物量向根茎的分配比例显著降低, 向叶片和根系的分配比例显著提高。 羊草种群的相对密度和相对生物量也随着氮素梯度的增加而显著提高。
\end{abstract}

关键词 典型草原 羊草 种群 生物量 资源分配 养分添加

\section{EFFECTS OF NITROGEN ADDITIONS ON A LEYMUS CHINENSIS POPULATION IN TYPICAL STEPPE OF INNER MONGOLIA}

\author{
PAN Qing-Min BAI Yong-Fei HAN Xing-Guo and Yang Jing-Cheng \\ ( Inner Mongolia Grassland Ecosystem Research Station, Institute of Botany, Chinese Academy of Sciences, Beijing 100093, China)
}

\begin{abstract}
Leymus chinensis, a rhizomatous graminoid, is a dominant species in the grasslands of northern China. The characteristics of $L$. chinensis populations have been well documented in many research papers. Because of overgrazing, grasslands of northern China have become degraded since the $1980 \mathrm{~s}$. As a result, the density and biomass of $L$. chinensis populations have decreased significantly. Fertilization is a common technique for management of pastures in many countries; however, it is not widely used in the grasslands of China. Nitrogen is an important driver of community succession in grassland ecosystems, but the response of $L$. chinensis populations to nitrogen additions in typical steppe, a semiarid area of northern China, remains unclear. We conducted a sequential nitrogen addition experiment in a lightly degraded grassland plot that was fenced in 1999. Nitrogen $\left(\mathrm{NH}_{4} \mathrm{NO}_{3}\right)$ was applied on July 5 for two years at application rates of: $0,1.75,5.25,10.5$, 17.5 , and $28 \mathrm{~g} \mathrm{~N} \cdot \mathrm{m}^{-2}$, respectively. There were 9 replicate $5 \mathrm{~m} \times 5 \mathrm{~m}$ plots of each of the six treatments with each plot spaced $1 \mathrm{~m}$ apart. A completely randomized design was used for this experiment. Before the experiment, soil samples were collected and dry bulk density, $\mathrm{pH}$, soil nitrogen and soil carbon were analyzed. After two years of nitrogen fertilization, we measured the density, height, aboveground biomass and belowground biomass of $L$. chinensis in each plot. The results showed that $L$. chinensis population characteristics were highly responsive to nitrogen additions. With an increase in nitrogen application rates, the density, height, aboveground biomass, belowground biomass and total biomass of $L$. chinensis increased significantly whereas the ratio of aboveground biomass/belowground biomass decreased. The allocation of biomass among plant parts was significantly affected by nitrogen additions: the proportion of biomass allocated to rhizomes decreased remarkably with increasing nitrogen rates whereas that allocated to leaves and roots increased significantly. The relative biomass and relative density of $L$. chinensis also increased with increasing nitrogen additions. In summary, adding nitrogen to lightly degraded grassland not only increased the density and biomass of $L$. chinensis population but changed the resource partitioning among plant parts as well.
\end{abstract}

Key words Typical steppe, Leymus chinensis, Population, Biomass, Resource allocation, Nitrogen fertilization 
于输入 (陈佐忠等, 1985)。近年来, 草原生态系统严 重退化, 其结构和功能显著受损, 草原生态系统的退 化机理及退化草地恢复途径的研究成为草地生态学 关注的热点之一(许志信等, 2000; 马兴旺和吕贻忠, 2000; 刘钟龄等, 2002)。在国外, 草地施肥是一项维 持草原生态系统养分平衡的常规管理措施 (Drake $e t$ $a l$, 1963; Ünlü et al., 1999)。在我国, 草地施肥尚 处在试验阶段, 在生产上未大面积应用。但最近的 研究结果显示, 草地施肥有助于草地生产力的恢复 和提高(程积民, 1997; 沈景林等, 1998)。

羊草( Leymus chinensis) 是多年生根茎禾草, 营养 繁殖能力很强, 具有典型的克隆生长特性, 是我国典 型草原群落的建群种或优势种, 在东北草原和内蒙 古草原经常形成大面积的单优种植被 (李建东, 1978)。因此羊草的种群生态学一直是我国草地生 态学者研究的热点之一。目前, 人们对羊草种群的 营养繁殖动态 (杨允菲和张宝田, 1992)、受养生长格 局(王昱生和盖晓春, 1995)、生物量生殖分配(王仁 忠, 2000)、密度制约机制(朴顺姬等, 1997) 等都进行 了深入研究。前人结果显示, 土壤微生境的养分状 况可以显著改变羊草无性系种群的生长格局 (王昱 生和盖晓春, 1995); 在松嫩平原单优羊草割草场, 施 用氮肥能增加羊草种群营养繁殖的数量 (杨允菲和 张宝田, 1992); 但是典型草原羊草种群对养分添加 的响应机制, 特别是在连续养分添加条件下羊草种 群的应答机理迄今未见报道。

为此, 我们在内蒙古典型草原区的天然草场设 计了一个系列的长期养分 (包括不同量和不同时期 的氮素、磷素和羊粪)添加试验。重点探讨连续养分 添加后的不同时期, 内蒙古典型草原植物种群特征 的变化。本文仅就不同梯度氮素对羊草种群的效应 进行了分析, 旨在揭示典型草原群落羊草种群对氮 素添加的响应机制, 为退化草地的恢复及草原管理 的肥料运筹提供依据。

\section{1 试验区自然概况与研究方法}

\section{1 试验区的自然概况}

试验区位于内蒙古锡林郭勒草原, 地处锡林河 南岸, 中国科学院内蒙古草原生态系统定位研究站 羊草样地的西侧。其地理坐标为 $43^{\circ} 32^{\prime} 45^{\prime \prime} \sim$ $43^{\circ} 33^{\prime} 10^{\prime \prime} \mathrm{N}, 116^{\circ} 40^{\prime} 30^{\prime \prime} \sim 116^{\circ} 40^{\prime} 50^{\prime \prime} \mathrm{E}$, 海拔约 $1224 \mathrm{~m}$ 。行政区划属内蒙古自治区锡林浩特市白 音锡勒牧场境内。该区域气候属半干旱草原气候, 冬季寒冷干燥, 夏季温和湿润。年平均温度为
$2.0{ }^{\circ} \mathrm{C}$; 年降水量 $350 \mathrm{~mm}$ 左右, 集中于 $6 \sim 9$ 月份, 占全年降水量的 $80 \%$ 左右。土壤类型为暗栗钙土。 试验区于 1999 年 10 月围封, 围封前属冷季放牧场, 按照刘钟龄等 (2002) 对退化草地的分类, 属于 I 度 退化的羊草草原。组成群落的植物种约 38 个, 以广 旱生根茎禾草羊草占优势, 其次为大针茅 (Stipa grandis)、冰草( Agropyron cristatum) 等旱生密丛禾草。 根据内蒙古草原生态系统定位研究站 25 年的长期 监测资料, 牧草一般在 4 月中、下旬返青, 8 月中、下 旬地上生物量达高峰, 9 月中、下旬枯黄。

\section{2 试验设计}

2000 年 4 月, 选择地势平坦、植被均匀的有代 表性的地段, 开始实施了长期养分添加试验。氮素 添加梯度试验共设计了 7 个处理, 为了保证氮素是 最主要的影响因子, 参照美国明尼苏达大学 Tilman 和 Wedin（1991）的草地氮素梯度试验设计, 对于其 中的 6 个处理,每年按表 1 施用磷素、钾素和微量元 素。6 个处理依次为: $\mathrm{NO}$ (不施用氮素, 不完全对 照); $\mathrm{N} 1.75$ (施用 $5 \mathrm{~g} \mathrm{NH} \mathrm{NO}_{3} \cdot \mathrm{m}^{-2}$ ), N5.25(施用 $15 \mathrm{~g}$

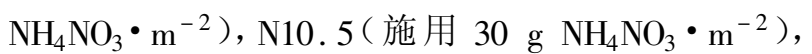
$\mathrm{N} 17.5$ (施用 $50 \mathrm{~g} \mathrm{NH} \mathrm{NHO}_{3} \cdot \mathrm{m}^{-2}$ )), N28(施用 $80 \mathrm{~g}$ $\mathrm{NH}_{4} \mathrm{NO}_{3} \cdot \mathrm{m}^{-2}$ )。同时设置了 1 个不施用任何营养元 素的处理 $\mathrm{ck}$ (完全对照)。试验小区面积为 $5 \mathrm{~m} \times 5$ $\mathrm{m}$, 间距为 $1 \mathrm{~m}$, 每个处理 9 次重复, 完全随机排列。 为了提高肥料的利用效率, 每年 7 月 5 日(该区域刚 进入雨季) 实施氮素梯度处理, 同一小区每年施用的 氮素梯度相同。表 1 中的营养元素先溶解为液体, 用微量喷雾器均匀地混合到洗涤干净烘干后过 60 目篮的细沙中, 将细沙再烘干, 施肥时, 按试验设计 的施用量和氮肥混匀后用手撒施。

表 1 试验中 $P$ 素、 $K$ 素和其它微量元素添加量

Table 1 Applied amount of phosphor, potassium and other elements

\begin{tabular}{ccc}
\hline $\begin{array}{c}\text { 添加元素 } \\
\text { Applied elements }\end{array}$ & $\begin{array}{c}\text { 添加量 } \\
\text { Applied amount }\left(\mathrm{g} \cdot \mathrm{m}^{-2}\right)\end{array}$ & $\begin{array}{c}\text { 溶液体积 } \\
\text { Solution volume }(\mathrm{ml})\end{array}$ \\
\hline $\mathrm{P}_{2} \mathrm{O}_{5}$ & 10 & 150 \\
$\mathrm{~K}_{2} \mathrm{O}$ & 10 & 150 \\
$\mathrm{MgSO}_{4}$ & 0.0625 & 200 \\
$\mathrm{ZnSO}_{4} \cdot 7 \mathrm{H}_{2} \mathrm{O}$ & 22.00 & 1000 \\
$\mathrm{MnCl}_{2} \cdot 4 \mathrm{H}_{2} \mathrm{O}$ & 180.0 & 1000 \\
$\mathrm{Na}_{2} \mathrm{MoO}_{4} \cdot 2 \mathrm{H}_{2} \mathrm{O}$ & 0.30 & 1000 \\
$\mathrm{H}_{3} \mathrm{BO}_{3}$ & 6.00 & 1000 \\
\hline
\end{tabular}

试验期间的降水情况如下: 2000 年降水量为 $314.1 \mathrm{~mm}$, 氮素施用前降水量为 $166.4 \mathrm{~mm}$, 氮素施 
用后降水量为 $147.7 \mathrm{~mm}$, 其中氮素施用后的一个月 内 $(7$ 月 5 日至 8 月 5 日)降水量为 $60.9 \mathrm{~mm}$ 。2001 年降水量为 $288.7 \mathrm{~mm}$, 氮素施用前降水量为 104.5 $\mathrm{mm}$, 氮素施用后降水量为 $184.2 \mathrm{~mm}$, 其中氮素施用 后的一个月内降水量为 $93.2 \mathrm{~mm}$ 。

试验实施前, 对试验地 $1 \mathrm{~m}$ 内不同土层的土壤 特性及养分含量进行了测定 (表 2)。其中, 土壤容 重采用环刀法测定, $\mathrm{pH}$ 值采用 $\mathrm{pH}$ 值电位法 (GB6920-86)测定,全氮含量采用半微量凯氏定氮法 测定, 全碳含量采用重铬酸钾-硫酸外加热法测定。

1.3 样品的采集与分析

典型草原羊草的根茎和根系主要集中在 $0 \sim 40$ $\mathrm{cm}$ 的土层中(姜恕等, 1985)。在连续两年养分添加 后, 为了全面了解氮素梯度对羊草种群地上和地下 构件的影响, 我们设计了规格为 $80 \mathrm{~cm} \times 60 \mathrm{~cm} \times 45$ $\mathrm{cm}$ (长 $\times$ 宽 $\times$ 高) 的钢板框 (上下底均不封堵, 相当 于样方框）。2001 年 8 月 25 日,在每小区内距东界 和南界各 $50 \mathrm{~cm}$ 处,将钢板框扣上, 期间注意保护牧 草, 用手帮助分开被压的牧草。用大锤将钢板框夯 入, 至钢板框地上部剩余 $5 \mathrm{~cm}$ 时, 用铁锨从侧面将 整个钢板框整体挖出, 运至冲根池。用自来水将土 壤从钢板框下端 (40 cm 土层处) 逐渐冲出。期间保 持植株完整，根据植株的地上部特征区分不同植株， 然后将不同植株的根系分开, 记载植物种类、各种植 物的分㯺(株从)数, 测量每个羊草分具的株高, 之后 再把羊草按构件(根系、根茎、茎、叶)分开, 于 $70{ }^{\circ} \mathrm{C}$ 下, 在恒温干燥箱中烘 $48 \mathrm{~h}$, 称干重。

有关指标的计算方法如下:

羊草种群地上生物量 $\left(\mathrm{g} \cdot \mathrm{m}^{-2}\right)=$ 钢板框内羊草 种群各地上构件生物量之和 $/(0.6 \times 0.8)$

羊草种群地下生物量 $\left(\mathrm{g} \cdot \mathrm{m}^{-2}\right)=$ 钢板框内羊草 种群各地下构件生物量之和 $/(0.6 \times 0.8)$

羊草种群密度 $\left(\right.$ tillers $\left.\cdot \mathrm{m}^{-2}\right)=$ 钢板框内羊草的
分蕃数 $/(0.6 \times 0.8)$

羊草种群生物量 $\left(\mathrm{g} \cdot \mathrm{m}^{-2}\right)=$ 羊草种群地上生物 量 + 羊草种群地下生物量

地下生物量 $/$ 地上生物量 $=$ 羊草种群地下生物 量/羊草种群地上生物量

羊草种群高度 $(\mathrm{cm})=$ 钢板框内全部羊草分菜 的高度之和/钢板框内羊草的分藥数

分鉴生物量 $\left(\mathrm{g} \cdot\right.$ tiller $\left.^{-1}\right)=($ 羊草种群地上生物 量 + 羊草种群地下生物量)/分蓝数

各构件生物量分配比例 $(\%)=$ 各构件生物量/ 羊草种群总生物量 $\times 100$

相对密度 $(\%)=$ 钢板框内羊草分藍数/钢板框 内所有植物种总分菜 $($ 株丛) 数 $\times 100$

相对生物量 $(\%)=$ 钢板框内羊草生物量/钢板 框内所有植物种总生物量 $\times 100$

\section{4 数据的处理与统计分析}

采用 SAS 软件对各指标进行单因素方差分析 (ANOVA), 用邓肯多重比较 (Duncan's multiple-range test) 检验在方差分析中有显著差异的变量间的差异 显著性。

\section{2 结果与分析}

\section{1 氮素对羊草种群密度和种群高度的影响}

羊草是典型的克隆植物, 构件数量的增加主要 依靠营养繁殖。在内蒙古典型草原轻度退化的冷季 放牧场, 连续两年实施氮素添加处理, 显著地改变了 羊草种群密度 (表 3), 所有添加氮素的处理, 羊草种 群的密度均显著高于两个对照处理。不同氮素梯度 处理之间, 羊草种群密度的差异均达显著水平, 随着 氮素添加量的增加, 羊草种群密度呈现显著增加的 趋势, 氮素添加量最多的 N28 处理, 羊草种群密度 较完全对照 (ck)增加了 1.84 倍。这说明, 连续两年 的氮素添加, 促进了羊草种群的营养繁殖过程羊草

表 2 试验地施肥前土壤特性

Table 2 Soil properties of experimental site before fertilization

\begin{tabular}{ccccc}
\hline $\begin{array}{c}\text { 土壤深度 } \\
\text { Soil depth }(\mathrm{cm})\end{array}$ & $\begin{array}{c}\mathrm{pH} \text { 值 } \\
\mathrm{pH}\left(\text { in H} \mathrm{H}_{2} \mathrm{O}\right)\end{array}$ & $\begin{array}{c}\text { 土壤容重 } \\
\text { Dry bulk density }\left(\mathrm{g}^{\bullet} \mathrm{cm}^{-3}\right)\end{array}$ & $\begin{array}{c}\text { 土壤全氮 } \\
\text { Total soil N }\left(\mathrm{g}^{\bullet} \mathrm{kg}^{-1}\right)\end{array}$ & $\begin{array}{c}\text { 土壤全碳 } \\
\mathrm{Total} \mathrm{soil} \mathrm{C}\left(\mathrm{g} \bullet \mathrm{kg}^{-1}\right)\end{array}$ \\
\hline $0 \sim 10$ & 7.79 & 1.30 & 0.91 & 12 \\
$10 \sim 20$ & 7.72 & 1.29 & 0.73 & 16.86 \\
$20 \sim 40$ & 7.84 & 1.32 & 0.76 & 13.21 \\
$40 \sim 60$ & 7.83 & 1.33 & 0.31 & 5.66 \\
$60 \sim 100$ & 7.81 & 1.34 & & \\
\hline
\end{tabular}


表 3 不同氮素梯度下羊草种群密度和高度 (平均值 \pm 标准差) Table 3 Density and height of Leymus chinensis population in different nitrogen gradations (Mean $\pm S D$ )

\begin{tabular}{ccc}
\hline $\begin{array}{c}\text { 氮素梯度 } \\
\text { Nitrogen gradation } \\
\left(\mathrm{g} \mathrm{N} \cdot \mathrm{m}^{-2}\right)\end{array}$ & $\begin{array}{c}\text { 密度 } \\
\text { Density }\left(\text { tillers } \mathrm{m}^{-2}\right)\end{array}$ & $\begin{array}{c}\text { 高度 } \\
\text { Height }(\mathrm{cm})\end{array}$ \\
\hline 对照 $\mathrm{ck}$ & $110.3 \pm 17.3^{\mathrm{e}}$ & $28.8 \pm 3.2^{\mathrm{c}}$ \\
0 & $127.7 \pm 13.7^{\mathrm{e}}$ & $27.5 \pm 4.1 \mathrm{c}$ \\
5.25 & $162.3 \pm 12.9^{\mathrm{d}}$ & $34.5 \pm 3.4^{\mathrm{b}}$ \\
10.5 & $222.1 \pm 14.1^{\mathrm{c}}$ & $36.0 \pm 1.8^{\mathrm{b}}$ \\
17.5 & $278.6 \pm 18.6^{\mathrm{b}}$ & $41.5 \pm 3.6^{\mathrm{a}}$ \\
28 & $313.3 \pm 20.2^{\mathrm{a}}$ & $42.7 \pm 2.3^{\mathrm{a}}$ \\
\hline
\end{tabular}

同一列数据中字母不同者表示数值间差异显著 $(p<0.05)$ Data with different letters are significantly different $(p<0.05)$ in the same column

的分菜数迅速增加。氮素添加对羊草种群的高度亦 有显著影响。所有添加氮素的处理, 羊草种群的高 度均显著高于两个对照处理。不同氮素梯度下, 尽 管 N5.25 处理和 N10.5 处理之间差异不显著、N17.5 处理和 N28 处理之间差异不显著, 但是从所有氮素 梯度处理看, 羊草种群的高度仍表现为随氮素梯度 的增加而逐渐增加的趋势。由此可见, 在较高的氮 素营养水平下, 羊草分藥平均高度将有所增加。

2.2 氮素对羊草种群生物量和地下生物量/地上生 物量比值影响

在内蒙古典型草原连续两年添加氮素, 不仅显 著地促进了羊草种群地上生物量的提高, 而且显著 地促进了地下生物量的提高 (表 4)。不同梯度的氮 素处理之间, 羊草种群总生物量、地上生物量和地下 生物量的差异均达到极显著水平; 随着氮素梯度的 增加, 羊草种群的总生物量、地上生物量和地下生物 量均呈现显著增加的趋势。在所有添加氮素的处理 中, 最高氮素添加量的处理 N28 获得了最高的总生 物量、地上生物量和地下生物量。与完全对照 ( ck) 相比较, 该处理总生物量增加了 $175.9 \mathrm{~g} \cdot \mathrm{m}^{-2}$, 其中, 地下生物量增加 $102.6 \mathrm{~g} \cdot \mathrm{m}^{-2}$, 地上生物量增加了 $73.3 \mathrm{~g} \cdot \mathrm{m}^{-2}$; 尽管地下生物量的绝对增加量显著大 于地上生物量的增加量, 但是从相对增加量看, 该处 理羊草种群总生物量提高了 $86.84 \%$, 地下生物量 提高了 $65.77 \%$, 而地上生物量提高了 $194.69 \%$, 地 上生物量的相对增幅显著高于地下生物量的相对增 幅。其它添加氮素的处理也表现出相同的趋势。由 此可见, 在典型草原施用氮肥, 不仅对羊草种群地下 生物量有促进效应, 而且对地上生物量的调节效应 更为显著。

地下生物量/地上生物量比值一方面反映了植
物地上和地下构件之间的关系, 另一方面反映了植 物对自身资源的空间配置状况。表 4 示出, 氮素添 加后显著改变了羊草种群的地下生物量/地上生物 量比值。与两个对照相比较, 所有添加氮素的处理 羊草种群的地下生物量/地上生物量比值均显著降 低。尽管 N 5.25 与 N10.5 处理差异不显著、N17.5 与 N28 处理差异不显著, 但是从全部氮素梯度处理 看, 随着氮素添加梯度的增加, 羊草种群的地下生物 量/地上生物量比值呈明显的降低趋势。与完全对 照 (ck) 相比较, 最高氮素添加量的 N28 处理的地下 生物量/地上生物量比值降低了 $43.75 \%$ 。可见, 氮 素添加对羊草种群地上和地下的关系具有显著的调 节效应。地下生物量/地上生物量比值的降低,一方 面, 标志着相同质量的地下构件负载地上构件的能 力显著提高; 另一方面, 表明羊草种群把自身更多的 资源分配到了地上构件中。

\section{3 氮素对羊草种群生物量分配的影响}

由表 5 可以看出, 在内蒙古典型草原, 羊草种群 生物量的分配比例为: 根茎 $>$ 根系 $>$ 茎 + 叶鞘 $>$ 叶 片。其中根茎生物量在总生物量中占的比例最大, 超过 60\%。氮素添加对羊草种群生物量分配比例 有显著影响。所有添加氮素的处理, 羊草种群根茎 生物量的分配比例均显著低于对照。尽管羊草种群 根茎生物量分配比例在 N5.25 处理与 N10.5 处理之 间差异不显著、在 N17.5 处理和 N28 处理之间差异 不显著, 但是从总体趋势看, 随着氮素梯度的增加, 羊草种群向根茎分配的生物量逐渐降低。氮素添加 量最高的 N28 处理根茎生物量分配比例最低, 比完 全对照降低了 $17.73 \%$ 。叶片是植物的光合同化器 官, 所有添加氮素的处理, 羊草种群叶片生物量在总 生物量中占的比例均显著高于两个对照, 随着氮素 梯度的增加, 羊草种群向叶片分配的生物量呈现逐 渐增加的趋势。氮素添加量最高的 N28 处理叶片 生物量分配比例最高, 比完全对照提高了 $77.18 \%$ 。 根系是羊草种群养分和水分的吸收器官, 氮素添加 后, 根系生物量分配比例表现出与叶片相同的趋势。 茎和叶鞘是植物主要的支撑器官, 氮素添加量较低 的 N5.25 处理和 N10.5 处理茎和叶鞘的生物量分配 比例与对照差异不显著, 但是氮素添加量较高的 N17.5 处理和 N28 处理茎和叶鞘的生物量分配比例 显著高于对照。这表明, 在内蒙古典型草原, 氮素添 加后随着土壤氮素营养的改善, 羊草种群在资源分 配策略上提高了向光合同化器官(叶片)和地下的养 
表 4 不同氮素梯度下羊草种群生物量和地下生物量/地上生物量比值 (平均值 \pm 标准差)

Table 4 Biomass distribution and the ratio of belowground biomass/aboveground biomass of Leymus chinensis population in different nitrogen gradations $($ Mean $\pm S D)$

\begin{tabular}{ccccc}
\hline $\begin{array}{c}\text { 氮素梯度 } \\
\text { Nitrogen gradation } \\
\left(\mathrm{g} \mathrm{N} \cdot \mathrm{m}^{-2}\right)\end{array}$ & $\begin{array}{c}\text { 总生物量 } \\
\text { Biomass } \\
\left(\mathrm{g}^{\bullet} \mathrm{m}^{-2}\right)\end{array}$ & $\begin{array}{c}\text { 地上生物量 } \\
\text { Aboveground biomass } \\
\left(\mathrm{g}^{\bullet} \mathrm{m}^{-2}\right)\end{array}$ & $\begin{array}{c}\text { 地下生物量 } \\
\text { Belowground biomass } \\
\left(\mathrm{g}^{\bullet} \mathrm{m}^{-2}\right)\end{array}$ & $\begin{array}{c}\text { 地下生物量/地上生物量比值 } \\
\text { Belowground biomass/ } \\
\text { Aboveground biomass }\end{array}$ \\
\hline $\begin{array}{c}\text { 对照 } \mathrm{ck} \\
0\end{array}$ & $276.6 \pm 13.2^{\mathrm{e}}$ & $45.2 \pm 8.5^{\mathrm{e}}$ & $231.4 \pm 8.0^{\mathrm{e}}$ & $5.12 \pm 0.10^{\mathrm{a}}$ \\
5.25 & $280.4 \pm 10.5^{\mathrm{e}}$ & $46.5 \pm 6.3^{\mathrm{e}}$ & $233.9 \pm 10.7^{\mathrm{e}}$ & $5.03 \pm 0.31^{\mathrm{a}}$ \\
10.5 & $332.2 \pm 10.2^{\mathrm{d}}$ & $63.5 \pm 7.6^{\mathrm{d}}$ & $268.7 \pm 6.9^{\mathrm{d}}$ & $4.23 \pm 0.25^{\mathrm{b}}$ \\
17.5 & $417.7 \pm 12.3^{\mathrm{c}}$ & $82.4 \pm 8.4^{\mathrm{c}}$ & $335.3 \pm 6.4^{\mathrm{c}}$ & $4.07 \pm 0.17^{\mathrm{b}}$ \\
28 & $469.4 \pm 15.6^{\mathrm{b}}$ & $118.5 \pm 8.2^{\mathrm{b}}$ & $350.9 \pm 11.1^{\mathrm{b}}$ & $3.13 \pm 0.11^{\mathrm{c}}$ \\
\hline
\end{tabular}

同一列数据中字母不同者表示数值间差异显著 $(p<0.05)$ Data with different letters are significantly different $(p<0.05)$ in the same column

表 5 不同氮素梯度下羊草种群生物量分配(平均值 \pm 标准差)

Table 5 Biomass distribution of Leymus chinensis population in different nitrogen gradations (Mean $\pm S D$ )

\begin{tabular}{ccccc}
\hline $\begin{array}{c}\text { 氮素梯度 } \\
\text { Nitrogen gradation }\left(\mathrm{g} \mathrm{N} \cdot \mathrm{m}^{-2}\right)\end{array}$ & $\begin{array}{c}\text { 根茎 } \\
\text { Rhizomes }(\%)\end{array}$ & $\begin{array}{c}\text { 根系 } \\
\text { Roots }(\%)\end{array}$ & $\begin{array}{c}\text { 茎 }+ \text { 叶鞘 } \\
\text { Stems and sheaths }(\%)\end{array}$ & $\begin{array}{c}\text { 叶 } \\
\text { Leaves }(\%)\end{array}$ \\
\hline 对照 $\mathrm{ck}$ & $73.15 \pm 1.11^{\mathrm{a}}$ & $10.51 \pm 0.29^{\mathrm{c}}$ & $9.33 \pm 0.33^{\mathrm{b}}$ & $7.01 \pm 0.54^{\mathrm{c}}$ \\
0 & $72.74 \pm 0.96^{\mathrm{a}}$ & $10.68 \pm 0.33^{\mathrm{c}}$ & $9.43 \pm 1.00^{\mathrm{b}}$ & $7.15 \pm 0.37^{\mathrm{c}}$ \\
5.25 & $68.30 \pm 0.45^{\mathrm{b}}$ & $12.42 \pm 0.51^{\mathrm{b}}$ & $9.96 \pm 0.32^{\mathrm{b}}$ & $9.32 \pm 0.49^{\mathrm{b}}$ \\
10.5 & $67.94 \pm 0.79^{\mathrm{b}}$ & $12.34 \pm 0.26^{\mathrm{b}}$ & $10.04 \pm 0.59^{\mathrm{b}}$ & $9.68 \pm 1.25^{\mathrm{b}}$ \\
17.5 & $61.23 \pm 1.26^{\mathrm{c}}$ & $14.46 \pm 0.40^{\mathrm{a}}$ & $12.76 \pm 0.77^{\mathrm{a}}$ & $11.55 \pm 1.03^{\mathrm{a}}$ \\
28 & $60.18 \pm 1.02^{\mathrm{c}}$ & $14.05 \pm 0.62^{\mathrm{a}}$ & $13.35 \pm 0.35^{\mathrm{a}}$ & $12.42 \pm 0.71^{\mathrm{a}}$ \\
\hline
\end{tabular}

同一列数据中字母不同者表示数值间差异显著 $(p<0.05)$ Data with different letters are significantly different $(p<0.05)$ in the same column

分吸收器官(根系)物质分配的比例, 而这种改变是 以降低向根茎的物质分配比例为代价的,当土壤氮 素营养条件进一步改善时, 随着叶片生物量的增加， 羊草种群进一步降低向根茎生物量分配的比例, 用 于增加支撑器官(茎和叶鞘)的生物量分配比例。

2.4 氮素对羊草分藮植株生物量的影响

羊草分葟植株生物量反映的是羊草单个分藍的 个体性状。由图 1 可以看出, 氮素添加后羊草分䔔 植株的生物量 (8 月 25 日取样)显著提高, 所有添加 氮素的处理,羊草分菜植株的生物量均显著高于对 照。尽管 N5.25 处理与 N10.5 处理之间差异不显 著、N17.5 处理与 N28 处理之间差异不显著, 但是从 总体趋势看, 随着氮素添加梯度的增加, 羊草分䡞植 株的平均生物量呈逐渐增加的趋势。表明氮素添加 对羊草分藮植株的生物量也有一定的促进效应。

\section{5 氮素对羊草种群相对密度和相对生物量的影响}

相对密度和相对生物量反映了植物种群在群落 中的地位。氮素添加后羊草种群在典型草原羊草群 落中的优势地位得到进一步巩固, 其相对密度和相 对生物量均显著增加 (表 6)。不同氮素添加梯度处 理之间,羊草种群的相对密度差异和相对生物量差 异均达到显著水平, 随着氮素添加梯度的增加, 羊草 种群相对密度和相对生物量均呈现显著增加的趋 势。氮素添加量最高的 N28 处理, 羊草种群的相对
密度和相对生物量均为最高, 分别比完全对照 ( $\mathrm{ck}$ ) 提高了 $63.58 \%$ 和 $48.31 \%$ 。由此可见,氮素添加 后, 由于土壤养分状况改善, 羊草种群的无性繁殖过 程得到促进, 羊草种群分藥数量迅速增加, 种群的个 体数量在群落中的优势进一步扩大, 同时由于羊草 分藍植株平均高度和分䓝植株生物量的增加, 羊草 种群生物量占群落生物量的比例也显著增加。

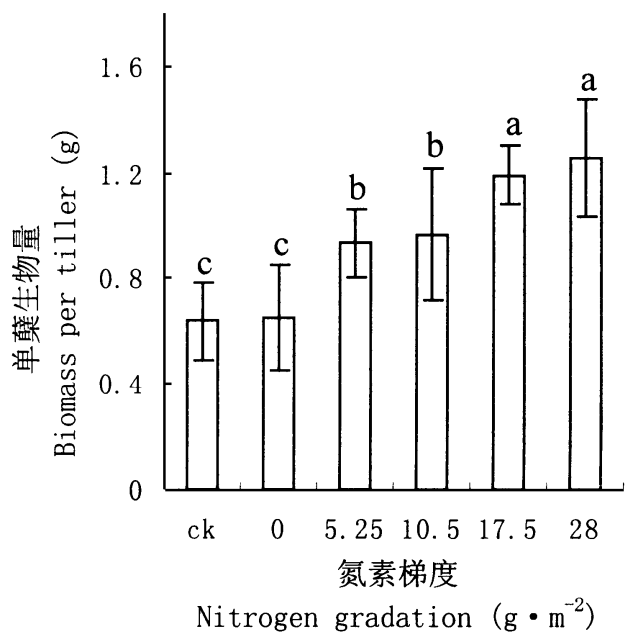

图 1 不同氮素梯度下羊草分葟植株生物量

Fig. 1 Tiller biomass of Leymus chinensis in different nitrogen gradations 具有不同字母的柱体间差异达显著水平 $(p<0.05)$ Bars with different letters are significantly different $(p<0.05)$ 
表 6 不同氮素梯度下羊草种群相对密度和 相对生物量 (平均值 \pm 标准差)

Table 6 Relative density and relative biomass of Leymus chinensis population in different nitrogen gradations $($ Mean $\pm S D)$

\begin{tabular}{ccc}
\hline $\begin{array}{c}\text { 氮素梯度 } \\
\text { Nitrogen gradation } \\
\left(\mathrm{g} \mathrm{N}^{-2} \mathrm{~m}^{-2}\right)\end{array}$ & $\begin{array}{c}\text { 相对密度 } \\
\text { Relative density }(\%)\end{array}$ & $\begin{array}{c}\text { 相对生物量 } \\
\text { Relative biomass }(\%)\end{array}$ \\
\hline 对照 $\mathrm{ck}$ & $30.5 \pm 2.3^{\mathrm{e}}$ & $28.5 \pm 1.8^{\mathrm{e}}$ \\
0 & $29.7 \pm 1.0^{\mathrm{e}}$ & $29.1 \pm 2.3^{\mathrm{e}}$ \\
5.25 & $36.6 \pm 1.6^{\mathrm{d}}$ & $34.9 \pm 1.2^{\mathrm{d}}$ \\
10.5 & $40.1 \pm 2.0^{\mathrm{c}}$ & $40.7 \pm 3.1^{\mathrm{c}}$ \\
17.5 & $47.3 \pm 1.2^{\mathrm{b}}$ & $49.1 \pm 1.7^{\mathrm{b}}$ \\
28 & $51.8 \pm 2.7^{\mathrm{a}}$ & $54.3 \pm 2.0^{\mathrm{a}}$ \\
\hline
\end{tabular}

同一列数据中字母不同者表示数值间差异显著 $(p<0.05) \quad \mathrm{Da}-$ ta with different letters are significantly $\operatorname{different}(p<0.05)$ in the same column

\section{3 讨 论}

羊草是内蒙古典型草原的群落建群种或优势 种。由于内蒙古典型草原长期超限度利用和不合理 的管理, 草原退化导致羊草种群密度和生物量显著 降低。轻度退化的羊草草原, 羊草种群密度降低 $28.3 \%$ 左右, 而严重退化的羊草草原, 羊草种群密度 降低幅度可达 94.5\%(刘钟龄等, 2002)。羊草种群 的消长可作为衡量羊草草原群落演替方向的重要指 标。通过两年的氮素添加, 羊草种群密度、高度、总 生物量、地上生物量和地下生物量均显著增加。相 关分析表明 (表 7), 羊草种群的密度、高度、总生物 量、地下生物量和地上生物量与氮素梯度均呈显著 正相关关系。说明轻度退化的羊草草原, 通过氮素 的添加可以促进羊草种群密度和生物量的提高, 从 而加速羊草种群的恢复。

前人研究证实, 土壤的营养资源可以调控无性 系草本根茎植物的受养行为 (Foraging behaviour), 当 植物受到资源丰富的生境时, 无性系植物的隔离者 长度 (Spacer length, 即相邻两个分株间根茎的长度) 会减少, 而分枝强度 (Branching intensity, 即每个分枝 产生隔离者的数目)会增加 (Sutherland \& Stillman, 1988; 王昱生和盖晓春, 1995)。杨允菲和张宝田 (1992)的研究表明,施肥对羊草种群的效应还表现 在羊草个体死亡的减少。本试验表明,氮素对羊草 种群的调节效应, 不仅表现在羊草种群密度 (表 3) 和生物量(表 4) 显著增加, 而且表现在羊草种群资 源的分配策略发生了改变。未添加氮素的对照处 理, 羊草根茎的生物量分配比例高达 $70 \%$ 以上(表 5）。随着氮素梯度的增加, 根茎的生物量分配比例 显著降低, 叶片和根系的生物量分配比例显著提高
（表 5)。叶片是光合器官, 是植物获取地上资源的 主要构件; 根系是养分、水分的吸收器官, 是植物获 取地下资源的主要构件。生物量分配比例的改变反 映了羊草种群对不同的土壤养分状况应答策略不 同,在土壤营养丰富的条件下,将更多的生物量分配 到获得外部资源的构件中, 有利于羊草种群的繁衍 和扩张。

表 7 羊草种群数量特征与氮素梯度的相关关系

Table 7 Quantitative characteristics of Leymus chinensis population in relation to nitrogen gradation

\begin{tabular}{lcc}
\hline \multicolumn{1}{c}{ Index } & \multicolumn{1}{c}{$\begin{array}{c}\text { 回归方程 } \\
\text { Regression equation }\end{array}$} & $\begin{array}{c}\text { 确定系数 } \\
R^{2}\end{array}$ \\
\hline $\begin{array}{l}\text { 密度 } \\
\text { Density }\left(\text { tillers } \cdot \mathrm{m}^{-2}\right)\end{array}$ & $Y=7.3590 x+127.26$ & 0.8750 \\
$\begin{array}{l}\text { 高度 } \\
\text { Height }(\mathrm{cm})\end{array}$ & $Y=0.5425 x+29.629$ & 0.6555 \\
$\begin{array}{l}\text { 总生物量 } \\
\text { Biomass }\left(\mathrm{g}^{\circ} \mathrm{m}^{-2}\right)\end{array}$ & $Y=8.9682 x+290.63$ & 0.8307 \\
$\begin{array}{l}\text { 地上生物量 } \\
\text { Aboveground biomass }\left(\mathrm{g} \cdot \mathrm{m}^{-2}\right)\end{array}$ & $Y=3.3294 x+47.562$ & 0.9671 \\
$\begin{array}{l}\text { 地下生物量 } \\
\text { Belowground biomass }\left(\mathrm{g} \cdot \mathrm{m}^{-2}\right)\end{array}$ & $Y=5.6388 x+243.07$ & 0.8180 \\
\hline
\end{tabular}

放牧影响下羊草种群的生殖生态学研究表明, 在由轻牧到极牧的放牧系列上, 随着放牧强度的增 加, 羊草种群根茎的生物量分配比例增加, 其它构件 的生物量分配比例下降(王仁忠, 2000)。本试验显 示, 对于轻度退化的草原群落添加氮素, 随着氮素梯 度的增加, 羊草种群根茎的分配比例显著降低, 其它 构件的生物量分配比例显著提高。两个试验的结果 看上去截然相反, 但它们却是对立统一的, 从正反两 个方面反映了羊草种群在不同演替进程中对环境因 子的应答机制。放牧梯度试验反映的是羊草种群在 退化演替序列上对环境的响应和适应策略, 而养分 添加梯度试验反映的是羊草种群在恢复演替序列上 对环境的响应和适应策略。由此可见, 在两个相反 方向的演替序列上, 羊草种群对资源分配采取的策 略也是截然相反的。

由于羊草是典型的根茎型无性系植物, 存在着 生理整合现象，所以在野外试验中, 确定适宜的小区 间距至关重要。但是, 关于草原生态系统长期野外 试验适宜的小区间距设置, 迄今未见报道。为此, 我 们参考了美国明尼苏达大学 Tilman 和 Wedin（1991） 等在草原生态系统实施的长期氮素添加试验的小区 间距设置, 设计的小区间距为 $1 \mathrm{~m}$ 。由于选择的试 
验区地势平坦, 连续两年添加氮素后, 不同处理之 间, 不仅不同土层土壤氮素含量差异显著(潘庆民 等, 2004), 而且羊草种群的密度、高度、地上生物量、 地下生物量等指标的差异均达到显著水平。因此, 初步认为在本试验条件下, 小区间距设置为 $1 \mathrm{~m}$ 是 可行的,不会因相邻梯度的处理之间差异不显著而 影响整体试验效果。

\section{参 考 文 献}

Cheng JM (程积民)，Jia HY (贾恒义)，Peng XL (彭祥林) (1997). Biomass of fertilized grassland communities. Acta Prataculturae Sinica (草业科学) , 6, 22-27. (in Chinese with Engilsh abstract)

Chen ZZ (陈佐忠), Sheng XW (盛修武), Yang ZG (杨宗贵), Huang DH (黄德华) (1985). The ecological effects of fertilization during rainy season on various types of steppe on the Xilin river valley, Inner Mongolia. In: Inner Mongolia Grassland Ecosystem Research Station, Chinese Academy of Sciences (中国 科学院内蒙古草原生态系统定位研究站) ed. Research on Grassland Ecosystem (草原生态系统研究). Science Press, Beijing, 225 - 232. (in Chinese with English abstract)

Drake M, Colby WG, Bredakis E (1963). Yield of orchard grass as influenced by rates of nitrogen and harvest management. Agronomy Journal, 55, 361-362.

Jiang S (姜恕), Qi QH (戚秋慧), Kong DZ (孔德珍)（1985）。 A comparative study on production of Aneurolepidium chinense and Stipa grandis steppe communities at Baiinsile livestock farm in Inner Mongolia region. In: Inner Mongolia Grassland Ecosystem Research Station, Chinese Academy of Sciences (中国科学 院内蒙古草原生态系统定位研究站) ed. Research on Grassland Ecosystem (草原生态系统研究). Science Press, Beijing, 12 - 23. (in Chinese with English abstract)

Li JD (李建东) (1978). Aneurolepidium chinense (Trin) Kitagawa grassland in China. Journal of Jilin Normal University (吉林师 范大学学报), 1, 145 - 159. (in Chinese with English abstract)

Liu ZL (刘钟龄), Wang W (王炜), Hao DY (郝敦元), Liang CZ (梁存柱) (2002). Probes on the degeneration and recovery succession mechanisms of Inner Mongolia steppe. Journal of Arid Land Resources and Environment (干早区资源与环境)，16, 84 - 91. (in Chinese with English abstract)
Ma XW (马兴旺), Lu YZ (吕贻忠) (2000). Influence of grassland degradation on combined humus in soil organic-mineral complexes. Journal of Arid Land Resources and Environment (干旱 区资源与环境), 14, 69-73. (in Chinese with English abstract)

Pan QM（潘庆民), Bai YF (白永飞), Han XG (韩兴国), Yang JC (杨景成) (2004). Studies on the fate of labeled nitrogen applied to Leymus chinensis community of typical steppe in Inner Mongolia grassland. Acta Phytoeclogica Sinica (植物生态 学报), 28,665-671. (in Chinese with English abstract)

Piao SJ (朴顺姬), Yang C (杨持), Huang SF (黄绍峰), Song MH (宋明华) (1997). Density and growth dynamics of Leymus chinensis population. Acta Phytoeclogica Sinica (植物生态学 报), 21, 60 - 66. (in Chinese with English abstract)

Shen JL (沈景林), Zhang GS (张光圣), Meng Y (梦杨), Hu WL (胡文良), Lian DW (连大伟) (1998). Experimental study on degenerated grassland improvement in Alpine area. Grassland of China (中国草地), (5), 46-49. (in Chinese with English abstract)

Sutherland WJ, Stillman RA (1988). The foraging tactics of plants. Oikos, 52, 39-244.

Tilman D, Wedin D (1991). Plant traits and resource reduction for five grasses growing on a nitrogen gradient. Ecology, 72, 685 700 .

Ünlü K, Özenirler G, Yurteri C (1999) . Nitrogen fertilizer leaching from cropped and irrigated sandy soil in central Turkey. European Journal of Science, 50, 609-620.

Wang RZ (王仁忠) (2000). Effect of grazing on reproduction in Leymus chinensis population. Chinese Journal of Applied Ecology (应用生态学报), 11, 399-402. (in Chinese with English abstract)

Wang YS (王昱生), Gai XC (盖晓春) (1995). Foraging growth patterns and resource allocations of clonal Leymus chinensis population in the Songnen steppe. Acta Phytoeclogica Sinica (植物生 态学报), 19, 293 - 301. (in Chinese with English abstract)

Xu ZX (许志信), Zhao ML (赵萌莉), Han GD (韩国栋)。 2000. Eco-environmental deterioration and strategies for preventing it in Inner Mongolia. Grassland of China (中国草地), (5), 59-63. (in Chinese with English abstract)

Yang YF (杨允菲), Zhang BT (张宝田) (1992). An analysis of seasonal variation of vegetative propagation and the relationships between biomass and population density of Aneurolepidium chinensis in Songnen plain of China. Acta Botanica Sinica (植物学 报), 34, 443-449. (in Chinese with English abstract) 